\title{
Significance of swimming and feeding currents for nutrient uptake in osmotrophic and interception- feeding flagellates
}

\author{
V. J. Langlois ${ }^{1,3, *}$, A. Andersen ${ }^{1}$, T. Bohr ${ }^{1}$, A.W. Visser ${ }^{2}$, T. Kiørboe ${ }^{2}$ \\ ${ }^{1}$ Department of Physics and Center for Fluid Dynamics, Technical University of Denmark, 2800 Kgs. Lyngby, Denmark \\ ${ }^{2}$ National Institute for Aquatic Resources, Technical University of Denmark, Kavalergården 6, 2920 Charlottenlund, Denmark \\ ${ }^{3}$ Present address: Laboratoire de Sciences de la Terre, Université Claude Bernard Lyon 1 -ENS Lyon- C.N.R.S., \\ 2 rue Raphaël Dubois, 69622 Villeurbanne cedex, France
}

\begin{abstract}
We introduce 2 simple models for the flow generated by a self-propelled flagellate: a sphere propelled by a cylindrical flagellum and one propelled by an external point force. We use these models to examine the role of advection in enhancing feeding rates in 3 situations: (1) osmotroph feeding on dissolved molecules, (2) interception feeding on non-motile prey particles, and (3) interception feeding on motile prey (such as bacteria). We show that the Sherwood number is close to unity for osmotrophic flagellates, as suggested by most previous models. However, a more correct representation of the flow field than that predicted by a naive sinking sphere model leads to substantially higher clearance rates for interception-feeding flagellates. We find that a short flagellum is favourable for interception feeding, but at the cost of an increase in the drag on the body of the swimming unicell. We finally demonstrate that prey motility significantly enhances prey encounter rates in interception-feeding flagellates and is, in fact, often much more important for food acquisition than the feeding current.
\end{abstract}

KEY WORDS: Flagellates · Nutrient uptake $\cdot$ Interception feeders $\cdot$ Motile prey

\section{INTRODUCTION}

Many unicellular planktonic organisms are nonmotile and depend on prey particles (molecules or organisms) to come to them. This applies to many osmotrophs, such as non-motile pelagic diatoms that depend on nutrient molecules to diffuse to their cell surface before they can be taken up. It also applies to some phagotrophic particle feeders, such as radiolarians and helioflagellates that feed on motile prey, e.g. bacteria, that are eaten upon interception by the predator cell body or pseudopodia that extend from the cell body. Depending on whether the prey motility is diffusive or ballistic at the scale of encounter, transport of prey to the collector cell scales with either the radius (diffusive) or the radius squared (ballistic) of the spherical collector cell (Taylor 1922, Visser \& Kiørboe 2006). This implies that the volume-specific potential nutrient uptake rate scales inversely with cell size squared (diffusive) or cell size (ballistic), thus putting severe constraints on cell size: only small cells are efficient diffusion feeders.

However, many if not most pelagic unicells are motile or generate a feeding current, and motility or feeding currents add an advective component to the transport of nutrient molecules and prey particles to the collector cell. It is normally assumed that phagotrophic flagellates that directly intercept prey particles (interception feeders) depend solely on the flow generated by the beating flagellum to clear the water for prey particles (Fenchel 1984, Shimeta \& Jumars (1991), although prey motility may be significant for prey encounter in some cases (Fenchel 1982, Shimeta 1993, Kiørboe 2008). In contrast, it is generally assumed that nutrient transport to bacteria and autotrophic flagellates depends almost entirely on molecular diffusion and that swimming and feeding currents play a negligible role (Kiørboe 1993, Karp-Boss et al. 1996). This is 
even more surprising given that the diffusivity of nutrient molecules is of a similar order to that of motile bacterial prey. The relative importance of advective and diffusive transport can be evaluated by the Péclet number:

$$
\mathrm{Pe}=\frac{V R}{D}
$$

where $V$ is the swimming velocity of the collector cell of radius $R$, and $D$ is the coefficient of diffusion. Motile bacteria typically have a $\mathrm{Pe}<<1$, which is consistent with the concept that advection plays a negligible role. For flagellates with a radius larger than $10 \mu \mathrm{m}$, however, the Péclet number is of the order of 1 or larger, and advective processes are thus of potential importance. The enhancement of the nutrient flux to the cell, $Q$, relative to the purely diffusive nutrient flux to the cell is measured by the Sherwood number:

$$
\mathrm{Sh}=\frac{Q}{4 \pi R D C_{\infty}}
$$

where $C_{\infty}$ is the nutrient concentration far away from the cell. For swimming osmotrophs, Karp-Boss et al. (1996) have assumed that the flow is well approximated by the flow around a sinking sphere at a low Reynolds number and found Sherwood numbers to be close to unity when the cell radius is less than $10 \mu \mathrm{m}$, i.e. that swimming does not help nutrient uptake.

The above conceptualisation of nutrient transport in motile unicells is based on simplified assumptions of the flow field around the swimming cells. For interception-feeding flagellates, one can assume that prey particles following streamlines that pass within 1 prey particle radius of the collector cell are intercepted and captured, and that the clearance rate thus becomes the volume flow rate of water through the annulus with an inner radius equal to the cell radius and outer radius equal to the cell radius plus the prey radius. Fenchel (1984) and Shimeta \& Jumars (1991) computed the clearance rate for a spherical collector assuming that the flow velocity is constant everywhere within the annulus and equal to the swimming velocity of the flagellate, thus ignoring the no-slip condition at the cell surface. Other assessments have assumed low Reynolds number flow around a translating sphere moved by a body force (sinking sphere) (Spielman 1977, Fenchel 1982, Kiørboe \& Titelman 1998), leading to clearance rates that not only differ in magnitude (they are smaller) from those of the above scenario but also in how they scale with sizes of prey and predator cells.

However, flagellates and other motile unicells are not moved by body force; they are self-propelled, i.e. pushed or pulled through the water by one or more beating flagella. This implies that streamlines come closer to the surface of the cell than in the case of a sinking cell, and that current models of clearance rates in interception-feeding flagellates and nutrient transport in swimming flagellates underestimate the role of advection. Advances from this situation were recently made by Magar (2003) and Magar \& Pedley (2005), who modelled a self-propelled microorganism as a squirmer - a virtual, spherical micro-organism that swims by moving its surface tangentially to itself. A ciliate with infinitesimally short cilia distributed all over its cell body is probably what comes closest to the squirmer, and a squirmer-like model has been successfully applied to spherical colonial flagellates (volvox) (Short et al. 2006, Solari et al. 2006). However, the squirmer is an unrealistic representation of a swimming flagellate. For the squirmer, the Sherwood numbers are on the order of 5 at Péclet numbers typical for medium sized flagellates ( 1 to $10 \mu \mathrm{m}$ ) (Magar et al. 2003), thus suggesting a significant enhancement of solute transport due to swimming. More dramatically, the clearance rate using the squirmer model turns out to be several orders of magnitude higher than for the sinking sphere. However, the squirmer likely overestimates the effects of swimming for a flagellate, because the no-slip condition on the cell surface is replaced by a slip condition with prescribed tangential velocity.

In the present study, we consider 2 simple models for the flow generated by a self-propelled flagellated microorganism: a sphere propelled either by a cylindrical flagellum or by an external point force. We use these models to examine the role of propulsion in enhancing nutrient acquisition in 3 situations: (1) osmotroph feeding on dissolved molecules, (2) interception feeding on non-motile prey particles, and (3) interception feeding on motile prey (such as bacteria). We present the 2 models and describe the properties of the flow created by the flagellate and make analytical estimates that allow us to derive a simple expression for the swimming speed. We study the influence of the flagellum on osmotroph feeding and show that the Sherwood number is close to unity for osmotrophic flagellates, as suggested by most previous models. We then examine the effect of interception feeding on passive prey and show that the specific properties of the flow induced by the propulsion lead to an enhancement of the nutrient uptake for the flagellate, and derive a simple estimate for the clearance rate using the expression for the swimming speed. We address the problem of feeding on motile bacteria by taking into account both the feeding current of the predator and the random motion of the prey. We show that the latter contributes for a large part to the prey encounter rate. Finally, our conclusions are discussed and compared to available experimental results. 


\section{MODELS}

\section{Sphere propelled by a cylindrical flagellum}

The body of the cell is approximated by a sphere of the radius $R$, and the effect of its flagellum is modelled by a thin cylinder of length $L$ and radius $W$ attached to the sphere (Fig. 1a). The cell can be either pushed or pulled by its flagellum. In the following, we chose a pulling flagellum. We note that, while the pushing or pulling flagellum will produce flow patterns that differ only in sign, the solution to the advection-diffusion problem is different for the two cases. A no-slip boundary condition is applied at the surface of the sphere. The effect of a beating flagellum is to drive fluid opposite to the swimming direction. If we neglect the timedependence and the detailed nature of the flow around the flagellum, we can model the propulsion by applying a given slip velocity, $V_{\mathrm{f}}$, at the surface of the cylinder, i.e. the surface of the cylinder acts as a conveyor belt. To avoid a discontinuity at the contact line between the sphere and the cylinder, a smooth step of the surface velocity is implemented: the velocity of the fluid along the flagellum is then:

$$
V_{\mathrm{s}}(z)=V_{\mathrm{f}}\left(1-\mathrm{e}^{-(z-R) / W}\right)
$$

where $z$ is the swimming direction, $z=0$ corresponding to the centre of the sphere. Our approach is different from most traditional flagellate models, since our aim is not to understand the details of how a flagellum generates thrust, but to understand how it influences the streamlines around the cell body, which are relevant for the nutrient uptake. For an overview of flagellate hydrodynamics, we refer to the seminal paper by Lighthill (1976).

a) swimming direction

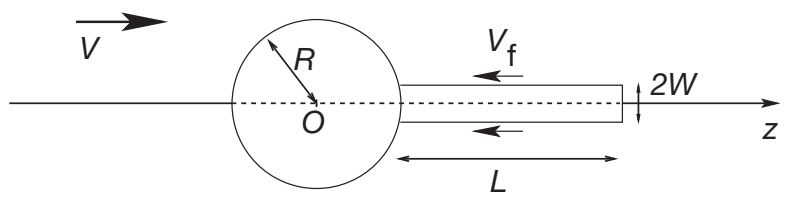

b) swimming direction

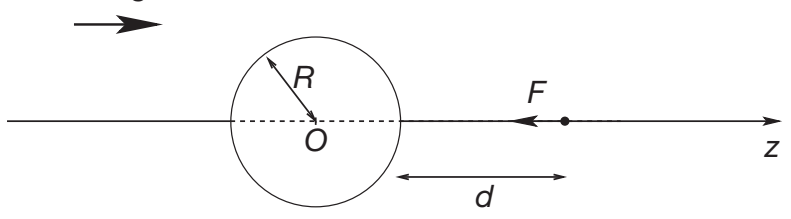

Fig. 1. (a) Sphere with no-slip condition propelled by a cylindrical flagellum with a prescribed surface velocity (the radius of the flagellum has been exaggerated for clarity); (b) sphere propelled by an external Stokeslet (point force). Swimming direction is to the right (the flagellum pulls the cell)
Flagellates are small enough for inertia to be completely negligible. The relative importance of inertial and viscous effects is expressed by the Reynolds number:

$$
\operatorname{Re}=\frac{\rho V R}{\eta}
$$

where $\rho$ is the mass density and $\eta$ is the viscosity of water. In the case of a flagellate of a radius $R=10 \mu \mathrm{m}$ and a swimming velocity $V=100 \mu \mathrm{m} \mathrm{s}^{-1}$, we estimate that $\mathrm{Re}=$ $10^{-3}$. Therefore, we can assume that the flow is described by the Stokes equation, which is valid in the limit of Re $<<1$, and the equation for incompressibility:

$$
\begin{aligned}
& \nabla p=\eta \nabla^{2} \boldsymbol{v} \\
& \nabla \cdot \boldsymbol{V}=0
\end{aligned}
$$

where $\boldsymbol{v}$ is the velocity field and $p$ is the pressure field. We use the commercial software Comsol (version 3.4) to solve this system of equations numerically around the model flagellate. The flagellate is thus placed at the centre of a cylindrical domain of a height of $200 \times R$ and a diameter of $200 \times R$. We solve the equations in the reference frame of the sphere and apply a uniform pressure on the boundaries of the domain.

\section{Sphere propelled by an external Stokeslet}

An alternative description of the flagellum is to consider its effect as a point force acting in front of the cell body, which we still represent as a sphere (Fig. 1b). The flow around a sphere generated by a point force external to the sphere at the distance $d$ from its surface was solved in the Stokes limit by Oseen (1927). When this flow is superimposed with that of a sinking sphere, we obtain a model which should be qualitatively similar to the cylindrical flagellum model when $d$ is equal to $L / 2$. The details of the analytical solution by Oseen (1927) have been presented by Pozrikidis (1992), and we give the solution adapted to our problem in Appendix 1.

\section{FLOWS AND FORCES}

\section{Flow structure}

The streamlines are front-back symmetric for the translating sphere moved by a body force (Fig. 2a). In contrast, the streamlines are front-back asymmetric for the 2 flagellate models, since the flagellum brings them closer to the surface of the sphere near the flagellum in both the model with the cylindrical flagellum (Fig. 2b) and the model with the external point force (Fig. 2c). With the point force the streamlines are pinched around the Stokeslet, where the velocity is 

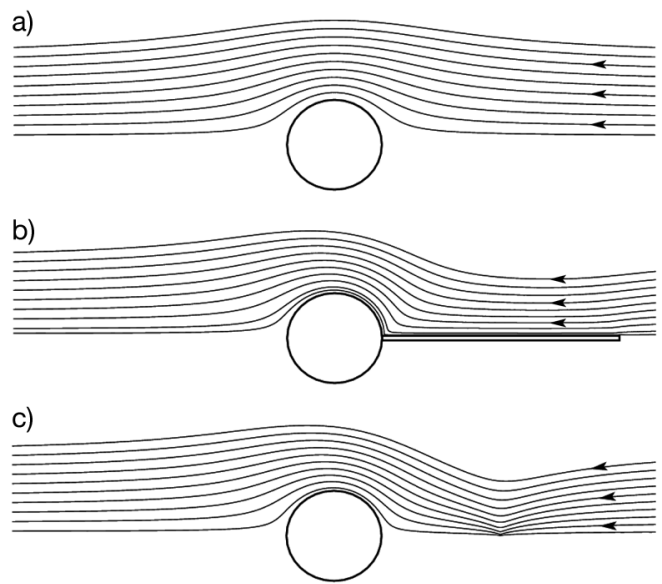

Fig. 2. Streamlines for the low Reynolds number flows created by the unicell models: (a) translating sphere moved by a body force; (b) sphere propelled by a cylindrical flagellum $(L=5 R)$; (c) sphere propelled by an external Stokeslet $(\mathrm{d}=2.5 R)$. Flow direction is from right to left

high. However, near the sphere the flow is less asymmetrical than with the cylindrical flagellum.

\section{Drag and thrust}

For the unicell to sustain a self-propelled motion at a constant speed $V$ the thrust, $F_{\mathrm{f}}$, produced by the flagellum has to balance the drag, $F_{\mathrm{S}}$ exerted by the fluid on the cell body. For the model with the cylindrical flagellum, the magnitude of these forces, $F$, can be calculated numerically from the solution of Eq. (5). We observe that, for a short flagellum, the force $F$ at a given speed $V$ is up to an order of magnitude larger than the drag, $6 \pi \eta R V$, on a sphere sinking at the same speed (Fig. 3). The force ratio decreases rapidly as the relative length of the flagellum increases, but remains greater than 2 for a flagellum length of up to 10 cell radii. This result suggests that flagellate models proposed in the literature (Christensen-Dalsgaard \& Fenchel 2003), in which the force generated by the flagellum balances the drag on a sinking sphere, should be used with care, since they can potentially underestimate the drag that has to be overcome to sustain motion by up to an order of magnitude.

We expect the drag on the cell $F_{\mathrm{s}}$ to be proportional to the radius $R$ and, due to the increased flow velocities near the flagellum (Fig. 2b), to depend both on the swimming speed and the local velocity imposed by the flagellum. A simple estimate can be written as the combination of the drag on a sinking sphere moving at the speed $V$ and that on a sinking sphere moving at the speed $V_{\mathrm{f}}$ :

$$
F_{\mathrm{s}}=6 \pi \eta R\left[(1-\alpha) V+\alpha V_{\mathrm{f}}\right]
$$

where $\alpha$ characterises the effect of the presence of the flagellum on the drag exerted on the sphere. This approach is reminiscent of that suggested by Lighthill (1976), where the drag on the cell body is calculated as the Stokes drag on a sphere moving with a velocity that includes an induced contribution from the flagellum evaluated at the centre of the sphere. Eq. (6) reduces to the normal Stokes drag on a sinking sphere when $\alpha=0$. We fitted the force obtained numerically by Eq. (6) and obtained the value $\alpha=0.13$ for a flagellum of width $W=0.01 \times R$ (Fig. 3). We find that the best fit captures the overall behaviour of the force, but slightly underestimates the force at intermediate values of flagellum length.

The slip velocity at the surface of the flagellum is $V_{\mathrm{f}}$ $-V$. Therefore, we can model the thrust produced by the flagellum by considering the force on a slender body translating lengthwise with a speed $V_{\mathrm{f}}-V$ at a low Reynolds number:

$$
F_{\mathrm{f}}=\frac{2 \pi \eta L\left(V_{\mathrm{f}}-V\right)}{\ln (L / W)-\beta}
$$

where $L$ is the length (major axis) and $2 W$ is the diameter (minor axis). For an ellipsoid, it is known that $\beta=$ 0.5 , and for a cylinder, which is less streamlined and therefore experiences a larger drag than a comparable ellipsoid, $\beta=0.81$ (Brennen \& Winet 1977). For the thrust provided by the flagellum in our numerical model, a satisfying fit is given by Eq. (7) with the shape factor $\beta=2.2$ (Fig. 3). The value of the constant is higher than for the translating cylinder, since the boundary conditions at both the free end of the flagel-

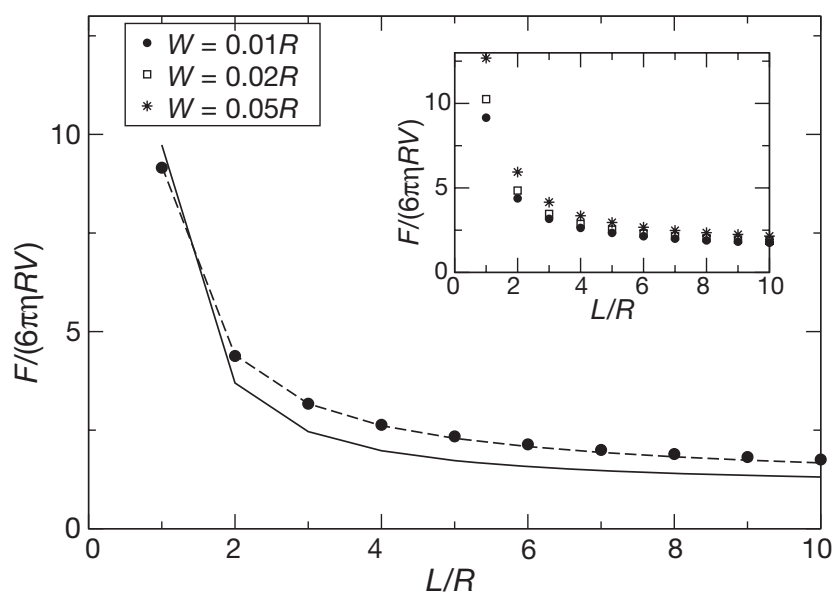

Fig. 3. Magnitude of the thrust exerted by the flagellum on the fluid as a function of the flagellum length. For a short flagellum, the thrust is up to an order of magnitude larger than the drag on a sphere sinking at a velocity equal to the swimming speed. It decreases with increasing flagellum length. Inset shows same quantity plotted for different values of the flagellum width $W$. Solid line shows the best fit of $F_{\mathrm{s}}$ using Eq. (6) and dashed line shows the best fit of $F_{\mathrm{f}}$ using Eq. (7) 
lum and the region where the flagellum is attached to the sphere make the flagellum less streamlined than the corresponding cylinder, therefore increasing the drag.

\section{Swimming velocity}

The swimming velocity, $V$, is in general smaller than the velocity imposed at the surface of the flagellum, $V_{\mathrm{f}}$ and when the length of the flagellum, $L$, becomes very large relative to the radius of the sphere, $R$, the swimming velocity slowly approaches the velocity imposed at the surface of the flagellum (Fig. 4). The simple models of the drag on the cell body (Eq. 6) and the thrust produced by the flagellum (Eq. 7) allow us now to estimate the swimming speed of the unicell as a function of the slip velocity of the flagellum. This can be obtained by equating the 2 force terms:

$$
V=\left\{1-\frac{3[\ln (L / W)-\beta]}{L / R+3(1-\alpha)[\ln (L / W)-\beta]}\right\} V_{\mathrm{f}}
$$

Eq. (8) with $\alpha=0.13$ and $\beta=2.2$ obtained in the 2 force fits (Fig. 3) correctly predicts the qualitative behaviour of the velocity ratio as function of the flagellum length (Fig. 4). Since the flagellum has to overcome drag on the sphere, this ratio is always smaller than 1 . The velocity ratio tends to 1 when the flagellum becomes very long, i.e. when the sphere is negligible compared with the flagellum.

\section{FEEDING BY DIFFUSION}

To examine the effect of advection on mass transport we next solve the advection-diffusion equation for the nutrient concentration $C$ :

$$
(\boldsymbol{v} \cdot \nabla) C=D \nabla^{2} C
$$

The equation is solved numerically with the software Comsol (version 3.4). The concentration of nutrients far away from the flagellate is fixed to $C_{\infty}$, and the spherical body is assumed to be a perfect absorber, i.e. $C(r=R)=0$. The influence of advection on the nutrient transport can be visualised by plotting $C$ for different values of Pe (Fig. 5). We vary Pe by varying $D$, while keeping $R$ and $V$ constant. The efficiency of swimming in improving the nutrient uptake is characterised by the Sherwood number defined in Eq. (2). From the numerical solution of the advection-diffusion equation, we integrate the flux through the sphere of radius $R$. The Sherwood number is plotted in Fig. 6 as a function of the Péclet number for

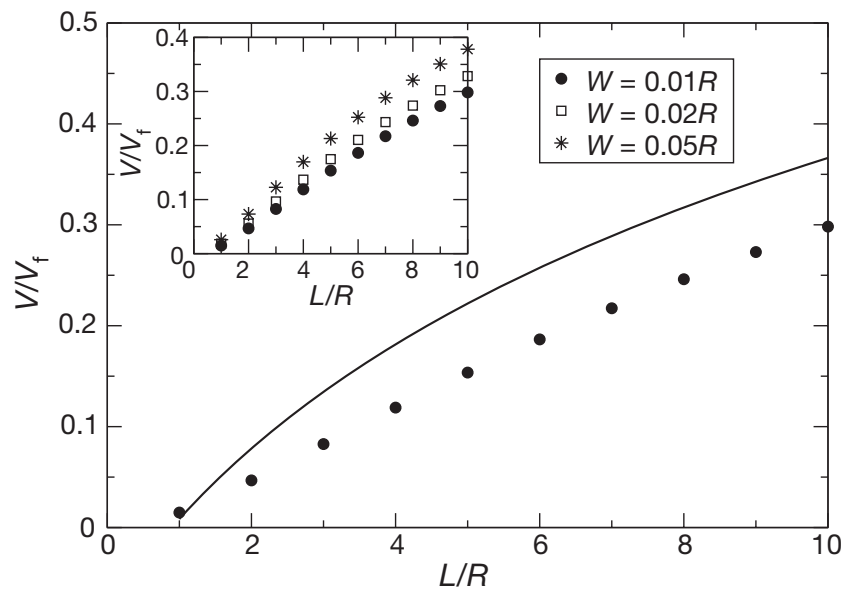

Fig. 4. Ratio between swimming speed $V$ and prescribed speed at the surface of the flagellum $V_{\mathrm{f}}$ as a function of the length $L$ of the flagellum relative to the radius of the cell $R$. Velocity ratio is less than 1 and increases slowly with increasing flagellum length. Inset shows velocity ratio $V / V_{\mathrm{f}}$ plotted for different values of the flagellum width $W$. Solid line shows Eq. (8) using $\alpha=0.13$ and $\beta=2.2$

different values of the flagellum length as well as for a sinking sphere for comparison. The asymmetry of the flow due to self-propulsion does not qualitatively modify the advection-diffusion process: in all cases, Sh tends to 1 for low values of $\mathrm{Pe}$ and varies like $\mathrm{Sh} \propto \mathrm{Pe}^{1 / 3}$ for high values of Pe. For all values of the Péclet number, the data for the sinking sphere are well fitted by the empirical formula by Clift et al. (1978):
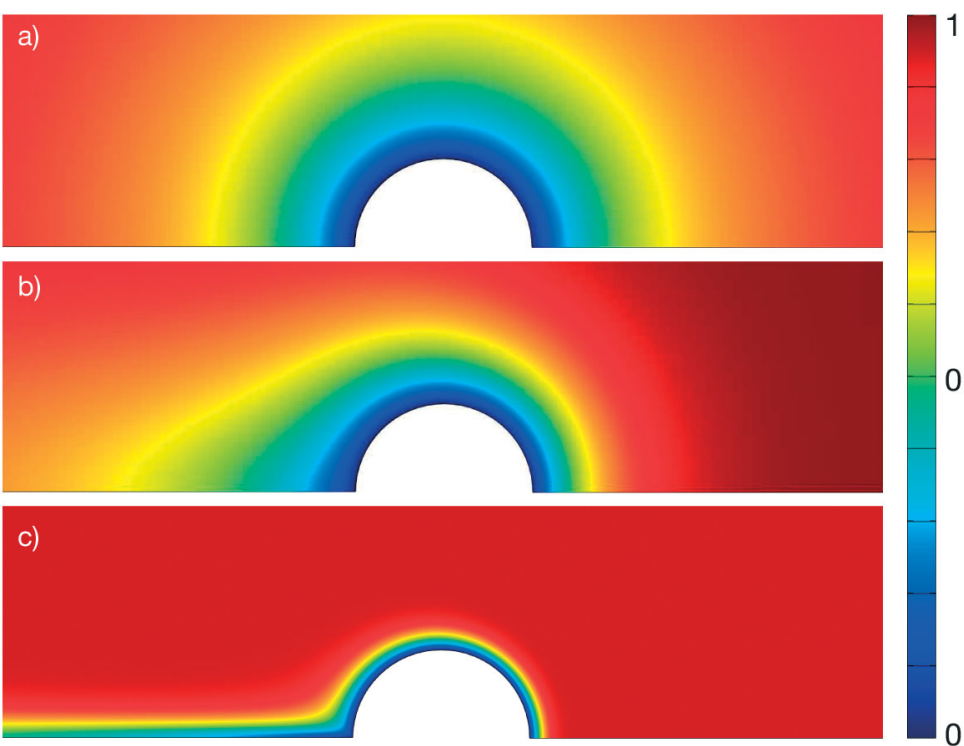

Fig. 5. Nutrient concentration $(C)$ around the flagellate with the cylindrical flagellum ( $L=5 R, W=0.01 R$, see Fig. 1 ) for different values of Pe (Péclet number): (a) $\mathrm{Pe}=10^{-2}$; (b) $\mathrm{Pe}=1$; (c) $\mathrm{Pe}=100$. Value of $C$ increases radially for small values of $\mathrm{Pe}$, whereas for large $\mathrm{Pe}$ a nutrient-depleted plume appears in the wake of the cell. Swimming direction is towards the right. Colour scale is normalised by the background concentration 


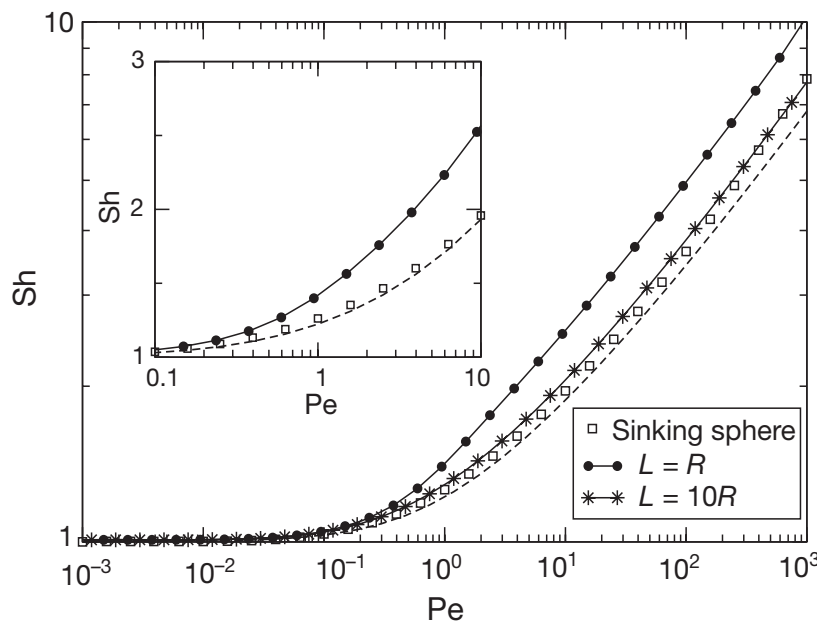

Fig. 6. Sherwood number (Sh) as a function of the Péclet number $(\mathrm{Pe})$ for the model flagellum with $W=0.01 R$, and $L=R$ and $L=10 R$ (see Fig. 1). For comparison, we show the result for a sinking sphere and the empirical formula given by Eq. (10) (dashed line). Inset shows a magnification of the intermediate Péclet number regime

$$
\mathrm{Sh}=\frac{1}{2}\left[1+(1+2 \mathrm{Pe})^{1 / 3}\right]
$$

The qualitative behaviour is similar for the model flagellate. Even for high Péclet numbers, self-propulsion only brings a small increase in nutrient uptake compared to the sinking sphere, e.g. at $\mathrm{Pe}=100$ we have $\mathrm{Sh}=3.6$ for the sinking sphere and $\mathrm{Sh}=4.9$ for the flagellate with $L=R$. However, in the case of most osmotrophs, the coefficient of diffusion $D$ is of the order of $10^{-5} \mathrm{~cm}^{2} \mathrm{~s}^{-1}$, which for flagellates of typical sizes and swimming speeds corresponds to Péclet numbers in the range of $10^{-1} \leqslant \mathrm{Pe} \leqslant 10$. Therefore, we are in the regime where $\mathrm{Sh} \leqslant 2.6$, and self-propulsion enhances nutrient uptake by less than a factor of 3 relative to purely diffusive nutrient uptake.

\section{FEEDING ON PASSIVE PARTICULATE PREY}

The clearance rate, $\Phi_{\mathrm{s}}$, for a sinking sphere can be calculated since the velocity field is known analytically. In this case, the clearance rate reads:

$$
\Phi_{\mathrm{s}}=\pi a^{2} V\left[1+\frac{1}{2}(1+a / R)^{-1}\right] \approx \frac{3}{2} \pi a^{2} V
$$

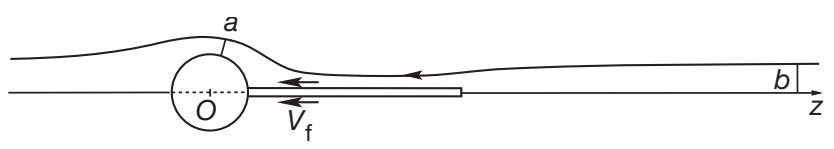

Fig. 7. Trajectory of a particle following a streamline in the neighbourhood of the swimming unicell. At infinity in both directions, the distance of the streamline to the $z$-axis is $b$. The minimal distance to the cell surface along the trajectory is $a$ where $a$ is the radius of the prey and where the approximate equality is valid when the prey is small in comparison with the predator (Spielman 1977, Kiørboe \& Titelman 1998). The clearance rate of the flagellate can be obtained numerically by computing the streamlines of the flow as illustrated in Fig. 7. Let us consider a particle far away upstream of the flagellate at a distance $b$ from the axis of symmetry. Assuming that the particle is passively transported by the flow, it will follow a streamline whose distance to the surface of the sphere reaches a minimum $a$. Thus, since the streamlines never cross, the clearance rate for a given particle size, $a$, is given by the integral:

$$
\Phi(a)=2 \pi \int_{0}^{b(a)} r v_{\mathrm{z}}\left(r, z_{\infty}\right) \mathrm{d} r
$$

where $r$ is the distance of the particle to the $z$-axis.

In the following, we present the clearance rates for the 2 different flagellate models. Due to the asymmetry of the flow, the volume scanned by the predator per unit time is greater for both models than that for the sinking sphere (Fig. 8). The length scales on the abscissas in Fig. 8a and Fig. 8b differ by a factor of 2 to emphasise the similarity between the clearance
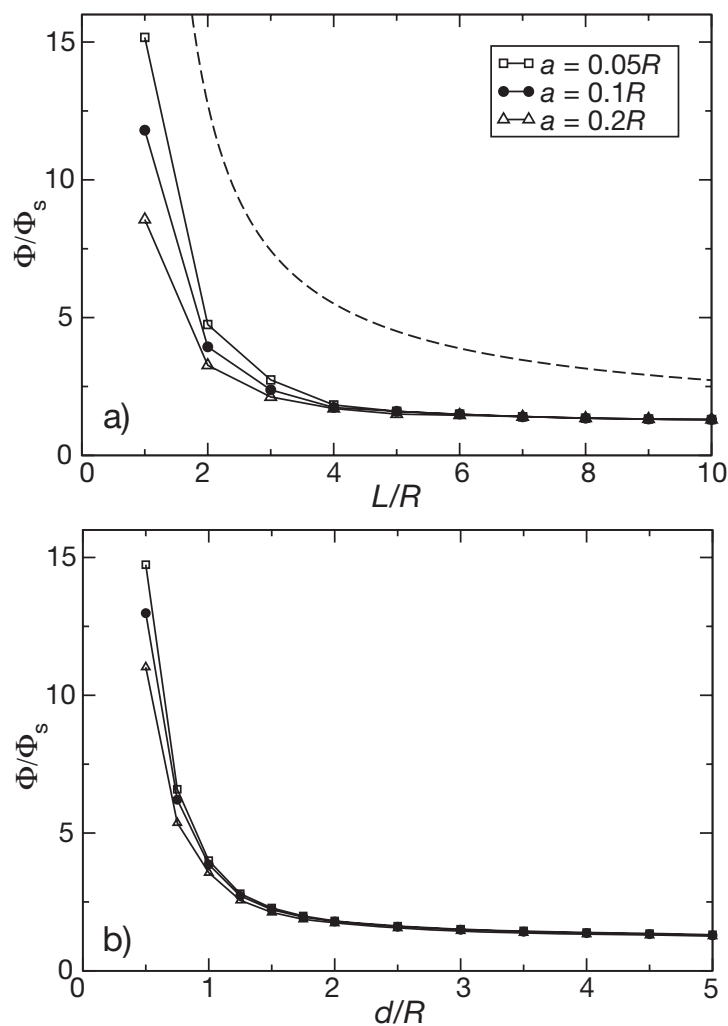

Fig. 8. Enhancement of the clearance rate $(\Phi)$ for prey size $a=$ $0.05 R, 0.1 R$ and $0.2 R$ for (a) a sphere propelled by a cylindrical flagellum of width $W=0.01 R$ as a function of its length $L_{i}$ dashed line represents the model given by Eq. (14) with $\alpha=$ 0.13 and $\beta=2.2$, and (b) a sphere propelled by an external Stokeslet as a function of the distance $d$ (see Fig. 1) 
rates for the 2 models when the distance $d$ is half the length of the flagellum $L / 2$. The enhancement in clearance rate is most significant for small prey and up to an order of magnitude for a short flagellum. The latter result can be understood by realising that, for a given swimming velocity, $V$, the flagellum surface velocity, $V_{\mathrm{f}}$, is highest for the shortest flagellum (Fig. 4). We can therefore understand this trend by assuming that the clearance rate for the flagellate can be modelled by an expression similar to the clearance rate for the sinking sphere in Eq. (11), but with the sinking speed replaced by the flagellum surface speed. As a simple approximation, we assume that the clearance rate is:

$$
\Phi=\frac{3}{2} \pi a^{2} V_{\mathrm{f}}
$$

and using Eq. (8) we estimate the clearance rate ratio:

$$
\frac{\Phi}{\Phi_{\mathrm{s}}}=\frac{V_{\mathrm{f}}}{V}=1+\frac{3[\ln (L / W)-\beta]}{L / R-3 \alpha[\ln (L / W)-\beta]}
$$

The estimate of the clearance rate ratio $\Phi / \Phi_{\mathrm{s}}$ decreases with increasing value of $L / R$, and with a very long flagellum the expression predicts that the clearance rate is not enhanced by the effect of the flagellum. Eq. (14), which is shown as the dashed line in Fig. 8a, therefore agrees qualitatively with the clearance rate obtained numerically, but overestimates it quantitatively since in Eq. (13), the velocity $V_{\mathrm{f}}$ is an upper limit of the speed of the flow past the cell body. The large enhancement of the clearance rate for the model flagellate with the short flagellum suggests that short flagella are favourable for interception feeding (Fig. 8a), but at the cost of an increase in the drag on the cell (Fig. 3), which suggests that long flagella are favourable for swimming.

\section{FEEDING ON MOTILE PREY}

For motile prey such as flagellated bacteria, relative motion is determined by the sum of the flow field generated by the predator, and the prey's own locomotion. We model the latter by a 3-dimensional random walk: in the absence of any external flow, a bacterium is supposed to follow a straight line at a velocity $\boldsymbol{v}_{\mathrm{p}}$ for a given time $\tau$ before randomly choosing a new orientation. Its mean free path is therefore $l_{p}=\tau v_{p}$.

The trajectory of a prey item is computed as follows: at the $\mathrm{n}$-th time-step $t_{\mathrm{n}}=n \tau$, the orientation of the prey velocity $\boldsymbol{v}_{\mathrm{p}}$ is chosen randomly (within a uniform distribution). Between $t_{\mathrm{n}}$ and $t_{\mathrm{n}+1}$, we integrate the equation of motion for the prey:

$$
\boldsymbol{r}\left(t_{\mathrm{n}+1}\right)=\boldsymbol{r}\left(t_{\mathrm{n}}\right)+\int_{t_{\mathrm{n}}}^{t_{\mathrm{n}+1}} \mathrm{~d} t\left[\boldsymbol{v}_{\mathrm{p}}+\boldsymbol{v}(\boldsymbol{r})\right]
$$

where $\boldsymbol{V}(\boldsymbol{r})$ is the velocity field created by the predator. At time-step $t_{n+1}=(n+1) \tau$, a new orientation for the prey velocity is chosen, and the procedure is iterated. As a first approximation, the velocity field created by the swimming predator is modelled as that of a sinking sphere. The asymmetry of the streamlines very close to the surface should not be of great influence, since the mean free path of the prey is typically much larger (10 times) than the radius of the predator. The prey is initially randomly distributed in the fluid around the predator. The clearance rate is computed as the rate at which prey enter the capture sphere of radius $R+a$ divided by prey concentration once the system has reached its steady state. Note that in this case, prey can hit the sphere perpendicular to the surface, contrary to what happens when they passively follow the streamlines. Therefore, the clearance rate does not tend towards zero when prey size a tends toward zero, which contributes to increasing the clearance rate for small prey dramatically (Fig. 9). For typical values of the prey and predator swimming velocities the predator can catch up to 100 times more prey organisms than if they were not motile.

\section{DISCUSSION AND CONCLUSION}

Mechanisms and, consequently, rates of nutrient acquisition are fundamental properties of an organism. It is, therefore, of interest to understand these mechanisms and, specifically, to understand how physics constrains nutrient acquisition rates and how these rates change with organism size. Nutrient acquisition in aquatic protists that swim and/or generate feeding currents are not completely understood. The models presented here represent an improvement relative to

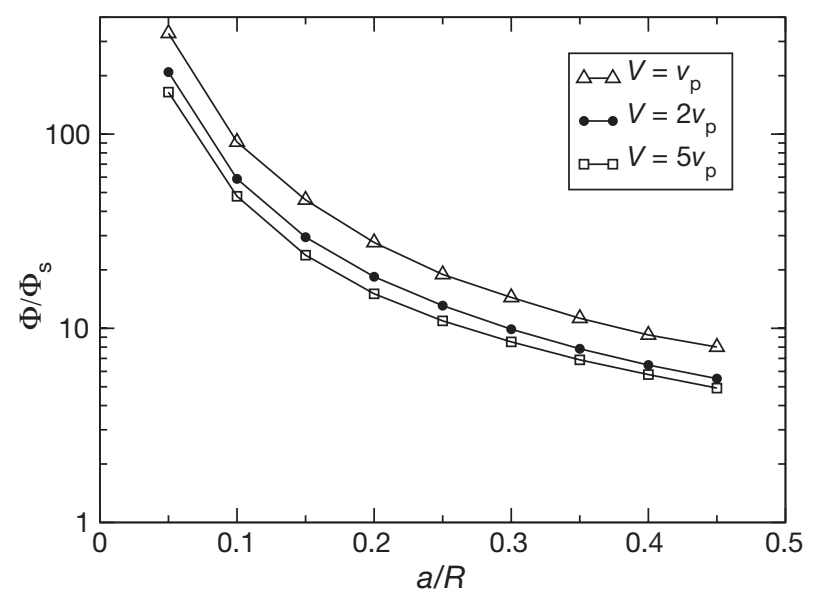

Fig. 9. Enhancement in the clearance rate $(\Phi)$ due to prey motility for different values of the swimming velocity of the predator $(V)$ relative to the prey velocity $v_{\mathrm{p}}$ and a fixed run duration for the prey $\tau=10 R / v_{p}$, i.e. a mean free path $l_{p}=10 R$ 
some current models of protist feeding since they consider the self-propelled nature of protist motility, but they are still very primitive relative to the complex flagellar motion and flows generated by real protists (see e.g. Christensen-Dalsgaard \& Fenchel 2004). Thus, we do not pretend to provide an accurate description of the flow field generated by flagellated protists and thus of their nutrient acquisition rate, but we argue that our models are helpful in exploring the significance of advection in both osmotrophic and particle-feeding flagellates.

Our results do not change the present understanding that mass transport in swimming osmotrophic flagellated protists is due mainly to diffusion. Even the largest and fastest-swimming flagellates have a Péclet number of less than 10, and consequently Sherwood numbers of less than about 2.6 (Fig. 6). Thus, swimming does not directly enhance nutrient uptake in small flagellated phytoplankters significantly; thus, they presumably swim in order to move. Swimming may allow phytoplankters to utilise chemical gradients to aggregate at sites of elevated nutrient availability, and thus indirectly improve nutrient acquisition (see e.g. Seymour et al. 2009).

If osmotrophic flagellates swim mainly to move, then our considerations suggest that they should possess relatively long flagella, since a long flagellum is energetically favourable for swimming. In contrast, interception-feeding flagellates may benefit somewhat from the feeding current generated by the flagellum and, hence, should have a relatively shorter flagellum, since feeding efficiency is inversely related to the length of the flagellum (Fig. 8). Actual lengths of flagella in naked flagellates provide only partial support for this prediction (see descriptions and pictures in Throndsen 1984). Thus, among the Chrysophyceae, most species appear to have flagellum lengths of 1 to 2 times their cell length, independent of whether they are autotrophic or heterotrophic (interception feeders). On the other hand, the longest flagella (up to 5 cell radii), are found among members of the purely autotrophic Prasinophyceae.

Many heterotrophic, particle-feeding pelagic flagellates posses various types of filtering structures through which water is passed and on which prey particles are retained. Our considerations do not apply to this feeding mode, but only to flagellates that directly intercept prey particles. This applies, for example, to Chrysomonads, which are pulled through the water by their undulating, hairy flagellum (see e.g. Fenchel 1986). The traditional description of the flow field generated by swimming flagellates, derived by portraying the flagellate as a sphere moved by a body force, suggests that interception feeding is a very inefficient process. As demonstrated in the present study, this description may somewhat underestimate the clearance rate of a self-propelled flagellate by maybe an order of magnitude, particularly for small prey and for flagellates with a short flagellum (Fig. 8). This is because the streamlines come much closer to the cell body for a self-propelled than for a sinking sphere.

How well does the model of a self-propelled flagellate predict actual measurements of clearance rates of non-motile prey particles? Monger \& Landry (1991) provided clearance estimates for the 3 to $8 \mu \mathrm{m}$ diameter interception feeding flagellate Paraphysomonas vestita, preying on 0.55 and $0.25 \mu \mathrm{m}$ radius plastic beads, of $5.7 \pm 0.2$ and $1.4 \pm 0.3 \mathrm{nl}$ flagellate $^{-1} \mathrm{~h}^{-1}$, respectively. These estimates are 6 to 7 times higher than those predicted by the sinking sphere model (see Eq. 11) applied with a swimming velocity of the flagellate of $V \simeq 175 \mu \mathrm{m} \mathrm{s}{ }^{-1}$ (Christensen-Dalsgaard \& Fenchel 2003). With a flagellum length of $L \simeq 10 \mu \mathrm{m} \simeq$ $3 R$, both of our models (propulsion by a cylindrical flagellum or an external Stokeslet) predict $\Phi \simeq 2 \Phi_{\mathrm{s}}$ for the corresponding values of a (Fig. 8), and thus a value that is closer to that observed but still too low by about a factor of 3 .

Monger \& Landry (1990) argued that such a 'geometric approach', where prey particles are assumed to strictly follow streamlines, is insufficient because repulsive hydrodynamic forces will tend to push prey particles away from streamlines in the direction away from the collector cell, while attractive surface forces (London-van der Waals forces) will pull prey particles closer to the collector cell. The balance between attractive and repulsive forces tends towards attraction the closer the streamlines come to the collector cell. Monger \& Landry (1990) showed that the geometric sinking sphere model may underestimate flagellate clearance rates by a magnitude similar to that found in the present study for the comparison with the self-propelled model. Because the self-propelled model brings streamlines closer to the collector than the sinking sphere model, the significance of attraction should be even more pronounced for the latter situation. Altogether, the 2 'corrections' combined may bring predicted clearance rates more in line with those estimated from experiments.

Our simulations suggest that feeding on motile prey particles may be much more efficient than feeding on non-motile particles by 1 to 2 orders of magnitude, and that prey motility is much more important for prey encounters than the feeding current in interception-feeding flagellates. Fenchel $(1982,1986)$ calculated that prey encounters by interception feeding in flagellates generating a feeding current and prey encounter due to motility of (bacterial) prey are about equally efficient (by describing prey motility using a diffusion analogy); however, Fenchel $(1982,1986)$ discounted the latter 
mechanism, because at that time it was generally believed that most pelagic bacteria were non-motile. It has since been shown that the majority of pelagic bacteria may in fact be highly motile (Fenchel 2001, Grossart et al. 2001). Fenchel (1982) and Kiørboe (2008) used a diffusion analogy to assess the effect of prey motility on encounter rates. However, such an approach will in most cases severely underestimate the effect of prey motility, because the run length (or mean free path) of motile bacteria is typically much larger than the size of the flagellate predator.

There is experimental evidence that prey motility is in fact the dominating mechanism for prey encounters in interception-feeding flagellates. Landry et al. (1991) showed that the clearance rate of Paraphysomonas vestita feeding on live Escherichia coli bacteria was about 20 times higher than when feeding on heatkilled E. coli. For characteristic parameters for the predator $\left(R=3 \mu \mathrm{m}, V=175 \mu \mathrm{m} \mathrm{s}^{-1}\right.$; Christensen-Dalsgaard \& Fenchel 2004) and prey $\left(v_{\mathrm{p}}=30 \mu \mathrm{m} \mathrm{s}^{-1}, \tau=1 \mathrm{~s}\right.$; Berg 1993), our simulations predict that the clearance rate should be about 10 times higher on the motile than on the heat-killed prey (Fig. 9). Landry et al. (1991) interpreted the difference in clearance rate as a result of active prey selection based on prey discrimination, while our simulations offer a simpler explanation.

In conclusion, the different roles for nutrient acquisition of swimming and feeding currents normally ascribed to phytoflagellates and interception-feeding flagellates in fact do not appear to be that different. Swimming does not significantly enhance delivery of solute nutrients to swimming unicellular osmotrophs, thus confirming the traditional view, but also often seem to play a secondary role for interception-feeding flagellates. This may not be that surprising after all, because motilities of bacteria and molecules are of similar magnitudes, as are the swimming speeds of autotrophic and heterotrophic flagellates.

\section{LITERATURE CITED}

Berg H (1993). Random walks in biology. Princeton University Press, Princeton, NJ

Brennen C, Winet H (1977) Fluid mechanics of propulsion by cilia and flagella. Annu Rev Fluid Mech 9:339-398

Christensen-Dalsgaard KK, Fenchel T (2003) Increased filtration efficiency of attached compared to free-swimming flagellates. Aquat Microb Ecol 33:77-86

Christensen-Dalsgaard KK, Fenchel T (2004) Complex flagellar motions and swimming patterns of the flagellates paraphysomonas vestita and pteridomonas danica. Protist 155: 79-87

Clift R, Grace J, Weber M (1978). Bubbles, drops, and particles. Dover Publications, Mineola, New York

Fenchel T (1982) Ecology of heterotrophic microflagellates. I. Some important forms and their functional morphology. Mar Ecol Prog Ser 8:211-223
Fenchel T (1984). Suspended marine bacteria as a food source. Plenum Press, New York

Fenchel T (1986) The ecology of heteretrophic microflagellates. Adv Microb Ecol 9:57-97

Fenchel T (2001) Eppur si muove: many water column bacteria are motile. Aquat Microb Ecol 24:197-201

Grossart H, Riemann L, Azam F (2001) Bacterial motility in the sea and its ecological implications. Adv Microb Ecol $25: 247-258$

Jiang H, Osborn T, Meneveau C (2002) The flow field around a freely swimming copepod in steady motion. Part I: theoretical analysis. J Plankton Res 24:167-189

Karp-Boss L, Boss E, Jumars P (1996) Nutrient fluxes to planktonic osmotrophs in the presence of fluid motion. Oceanogr Mar Biol Annu Rev 34:71-107

- Kiørboe T (1993) Turbulence, phytoplankton cell size and the structure of pelagic food webs. Adv Mar Biol 29:1-72

Kiørboe T (2008). A mechanistic approach to plankton ecology. Princeton University Press, Princeton, NJ

> Kiørboe T, Titelman J (1998) Feeding, prey selection and prey encounter mechanisms in the heterotrophic dinoflagellate Noctiluca scintillans. J Plankton Res 20:1615-1636

> Landry M, Lehner-Fournier J, Sundstrom J, Fagerness V, Selph K (1991) Discrimination between living and heatkilled prey by a marine zooflagellate, Paraphysomonas vestita (stokes). J Exp Mar Biol Ecol 146:139-151

> Lighthill J (1976) Flagellar hydrodynamics. SIAM Rev 18: $161-230$

> Magar V, Pedley T (2005) Average nutrient uptake by a selfpropelled unsteady squirmer. J Fluid Mech 539:93-112

> Magar V, Goto T, Pedley T (2003) Nutrient uptake by a selfpropelled steady squirmer. Q J Mech Appl Math 56:65-91

> Monger BC, Landry MR (1990) Direct-interception feeding by marine zooflagellates: the importance of surface and hydrodynamic forces. Mar Ecol Prog Ser 65:123-140

> Monger BC, Landry MR (1991) Prey-size dependency of grazing by free-living marine flagellates. Mar Ecol Prog Ser 74: $239-248$

Oseen C (1927). Neuere Methoden und Ergebnisse in der Hydrodynamik. Akademische Verlagsgesellschaft, Leipzig

Pozrikidis C (1992). Boundary integral and singularity methods for linearized viscous flow. Cambridge University Press

Seymour JR, Marcos, Stocker R (2009). Resource patch formation and exploitation throughout the marine microbial food web. Am Nat 173:E15-E29

Shimeta J (1993) Diffusional encounter of submicron particles and small cells by suspension feeders. Limnol Oceanogr 38:456-465

Shimeta J, Jumars P (1991) Physical mechanisms and rates of particle capture by suspension feeders. Oceanogr Mar Biol Annu Rev 29:191-257

Short MB, Solari CA, Ganguly S, Powers TR, Kessler JO, Goldstein RE (2006) Flows driven by flagella of multicellular organisms enhance long-range molecular transport. Proc Natl Acad Sci USA 103:8315-8319

Solari CA, Ganguly S, Kessler JO, Michod RE, Goldstein RE (2006) Multicellularity and the functional interdependence of motility and molecular transport. Proc Natl Acad Sci USA 103:1353-1358

Spielman LA (1977) Particle capture from low-speed laminar flows. Annu Rev Fluid Mech 9:297-319

Taylor GI (1922) Diffusion by continuous movements. Proc Lond Math Soc s2-20:196-212

Throndsen J (1984). The planktonic marine flagellates. Academic Press, San Diego, CA

> Visser AW, Kiørboe T (2006) Plankton motility patterns and encounter rates. Oecologia 148:538-546 
Appendix 1. Analytical solution for the Stokeslet model

We give the analytical solution of the hydrodynamic equations for the model presented in 'Sphere propelled by an external Stokeslet' in 'Models' (Oseen 1927, Pozrikidis 1992). The origin being defined as the centre of the sphere and $z$ as the swimming direction (Fig. 1b), $r$ designates the distance to the origin and $s$ is the distance to the $z$ axis: $s=\sqrt{x^{2}+y^{2}}$ and $r=$ $\sqrt{s^{2}+z^{2}}$. The position on the $z$-axis of the Stokeslet (point force) of strength $F$ is $\delta=R+d$ and the position of its image point is $C=R^{2} / \delta$. We need to introduce the distance to each of these points $r_{\delta}=\sqrt{s^{2}+(z-\delta)^{2}}$ and $r_{\mathrm{C}}=\sqrt{s^{2}+(z-c)^{2}}$. The Stokes equation being linear, the flow field created by a sphere propelled by a Stokeslet can be written as the sum of 2 terms: the Oseen flow field $\boldsymbol{v}^{\mathrm{O}}$, created by the point force with no-slip boundary conditions on the surface of the sphere and vanishing velocity at infinity; and the Stokes flow $\mathbf{v}^{\mathrm{S}}$, created by the motion of the sphere at a constant speed $V$ (Jiang et al. 2002). The radial and axial components of the velocity field thus read, respectively:

$$
\begin{aligned}
& v_{s}(s, z)=v_{s}^{\mathrm{S}}(s, z)+v_{s}^{\mathrm{O}}(s, z) \\
& v_{z}(s, z)=v_{z}^{\mathrm{S}}(s, z)+v_{z}^{\mathrm{O}}(s, z)
\end{aligned}
$$

where the Stokes flow around the sphere is given by

$$
\begin{aligned}
& V_{s}^{\mathrm{S}}(s, z)=s V \frac{3 R z\left(R^{2}-r^{2}\right)}{4 r^{5}} \\
& V_{Z}^{\mathrm{S}}(s, z)=V \frac{-R^{3}\left(s^{2}-2 z^{2}\right)+4 r^{3}\left(s^{2}+z^{2}\right)-3 R r^{2}\left(s^{2}+2 z^{2}\right)}{4 r^{5}}
\end{aligned}
$$

The flow created by the point force is

$$
\begin{aligned}
V_{S}^{\mathrm{O}}(s, z)= & s \frac{F}{8 \pi \eta}\left[\frac{z-\delta}{r_{\delta}^{3}}-\frac{R^{3}(z-C)}{\delta^{3} r_{C}^{3}}+\frac{R C\left(\delta^{2}-R^{2}\right)}{\delta^{3} r_{C}^{3}}-\frac{\left(r^{2}-R^{2}\right)\left(\delta^{2}-R^{2}\right)}{2 \delta^{3}} F_{s z}\right] \\
V_{z}^{\mathrm{O}}(s, z)= & \frac{F}{8 \pi \eta}\left[\frac{1}{r_{\delta}}+\frac{(z-\delta)^{2}}{r_{\delta}^{3}}-\frac{R}{\delta r_{C}}-\frac{R^{3}(z-C)^{2}}{\delta^{3} r_{C}^{3}}\right. \\
& -\frac{\delta^{2}-R^{2}}{\delta}\left(\frac{C^{2}}{R^{3} r_{C}}-\frac{2 R C(z-C)}{\delta^{2} r_{C}^{3}}+\frac{2 C^{3}(z-C)}{R^{3} r_{C}^{3}}\right) \\
& \left.-\frac{\left(r^{2}-R^{2}\right)\left(\delta^{2}-R^{2}\right)}{2 \delta^{3}} F_{z z}\right]
\end{aligned}
$$

with

$$
\begin{aligned}
& F_{\mathrm{Sz}}=-\frac{3 \delta}{R r_{C}^{3}}-3 R \frac{z-C}{r_{C}^{5}}+\frac{6 \delta c(z-C)}{R r_{C}^{5}}+\frac{3 R}{C} \frac{r_{C}^{2}+(z-c) C}{r_{C}^{3}\left(c r_{C}+z C-C^{2}\right)}-3 R \frac{r_{C}^{2}-(z-c) C+(z-2 c) r_{C}}{r_{C}^{2}\left(c r_{C}+z C-C^{2}\right)^{2}} \\
& F_{z z}=-\frac{3 \delta(z-C)}{R r_{C}^{3}}+\frac{R}{r_{C}^{3}}-\frac{3 R(z-c)^{2}}{r_{C}^{5}}-\frac{2 \delta c}{R r_{C}^{3}}+\frac{6 \delta(z-c)^{2} C}{R r_{C}^{5}}+\frac{3 R}{C} \frac{(z-c) r_{C}^{2}+(z-c)^{2} c+\left(r_{C}-c\right) r_{C}^{2}}{r_{C}^{3}\left(c r_{C}+z C-C^{2}\right)} \\
& -3 R \frac{\left[(z-C)+r_{C}\right]\left[r_{C}^{2}-(z-C) C+(z-2 c) r_{C}\right]}{r_{C}^{2}\left(C r_{C}+z C-C^{2}\right)^{2}}
\end{aligned}
$$

This flow created by the point force $F$ results in a drag on the sphere (Pozrikidis 1992)

$$
F_{\mathrm{O}}=\frac{F}{2}\left(\frac{3 R}{\delta}-\frac{R^{3}}{\delta^{3}}\right)
$$

Since the Stokeslet is supposed to sustain a motion at a constant speed $V$, the point force has to balance the total drag on the body (Jiang et al. 2002):

$$
F=6 \pi R \eta V+F_{\mathrm{O}}
$$

The strength of the Stokeslet must therefore be

$$
F=\frac{12 \pi R \eta V}{2-3 R / \delta+R^{3} / \delta^{3}}
$$

